\title{
SPECTROSCOPY OF GIANTS OF THE SAGITTARIUS DWARF GALAXY
}

\author{
P. BONIFACIO \\ Osservatorio Astronomico di Trieste \\ Via G.B. Tiepolo 11 I-34131 Trieste \\ L. PASQUINI \\ European Southern Observatory \\ K. Schwarzschild Strasse 2 D-85748 Garching bei Munchen - \\ Germany \\ P. MOLARO \\ Osservatorio Astronomico di Trieste \\ Via G.B. Tiepolo 11 I-34131 Trieste \\ AND \\ G. MARCONI \\ Osservatorio Astronomico di Roma \\ Via dell'Osservatorio 2, Monte Porzio Catone, I-00040 Roma, \\ Italy
}

\begin{abstract}
In this paper we present the first results of the analysis of intermediate resolution $(\Delta \lambda \sim 3.5 \AA)$ spectra of giants of the Sagittarius dwarf galaxy acquired using the ESO NTT telescope. From the deep CCD photometry of Marconi et al (1998a) we have selected a sample of giants representative of the metallicity spread suggested by the comparison of the colour-magnitude diagram of Sagittarius with those of galactic globular clusters. The spectra have been used to measure radial velocities, to confirm the membership to Sagittarius, and to provide a metallicity estimate by using spectral synthesis techniques. The analyzed stars show a spread in metallicities in the range $-1.0 \leq[\mathrm{Fe} / \mathrm{H}] \leq+0.7$, some 0.5 dex more metal-rich than the photometric estimates.
\end{abstract}




\section{Introduction}

The Sagittarius dwarf galaxy, discovered by Ibata et al (1994, 1995), at a distance of only $25 \mathrm{Kpc}$, offers a unique opportunity to study the stellar populations of an external galaxy. Comparison of the color-magnitude diagram of Sagittarius with those of Galactic globular clusters has suggested a spread in metallicity in the range $-0.7 \leq[\mathrm{Fe} / \mathrm{H}] \leq-1.6$ (Marconi et al 1998a). This may be the sign of a complex star-formation history characterized by several bursts. Because Sagittarius is some $16 \mathrm{Kpc}$ behind the Galactic Center there is confusion between Sagittarius and Bulge stars. Sagittarius revealed itself as a population with a mean radial heliocentric velocity around $140 \mathrm{kms}^{-1}$ and a small velocity dispersion, thus probable membership may be ascribed on the basis of the radial velocity. Intermediate resolution spectra allow to determine radial velocities with a precision around $20 \mathrm{kms}^{-1}$, sufficient to confirm membership. Moreover such low resolution spectra may be used to obtain a crude estimate of the metallicity which may be compared with the estimates based on the colour-magnitude diagrams. In this paper we report on radial velocities and abundances derived from grism spectra obtained with NTT+EMMI/MOS at ESO La Silla.

\section{Observations}

We used the multi-object-spectroscopy (MOS) mode of the EMMI instrument on the NTT $3.5 \mathrm{~m}$ telescope at ESO La Silla. The resolving power was about 1500, the usable spectral range was from about $480 \mathrm{~nm}$ to about 620 $\mathrm{nm}$. We acquired spectra of 57 stars, the log of the observations and further details may be found in Marconi et al (1998b).

TABLE 1. Abundances and atmospheric parameters

\begin{tabular}{cccccccc}
\hline$\#$ & $V_{0}$ & $\begin{array}{c}T_{\text {eff }} \\
\mathrm{K}\end{array}$ & $\log \mathrm{g}$ & $\begin{array}{l}{[\mathrm{Fe} / \mathrm{H}]} \\
\xi=2 \mathrm{kms}^{-1}\end{array}$ & $\begin{array}{c}{[\mathrm{Mg} / \mathrm{Fe}]} \\
\xi=2 \mathrm{kms}^{-1}\end{array}$ & $\begin{array}{c}{[\mathrm{Fe} / \mathrm{H}]} \\
\xi=1 \mathrm{kms}^{-1}\end{array}$ & $\begin{array}{c}{[\mathrm{Mg} / \mathrm{Fe}]} \\
\xi=1 \mathrm{kms}^{-1}\end{array}$ \\
\hline 105 & 17.55 & 5041 & 2.59 & -0.5 & +0.1 & -0.17 & -0.11 \\
115 & 17.84 & 4953 & 2.36 & -1.0 & +1.2 & -0.41 & +0.61 \\
124 & 17.00 & 4891 & 2.21 & -0.6 & +0.3 & -0.35 & +0.02 \\
128 & 17.40 & 4778 & 1.93 & +0.7 & -0.5 & +1.28 & -0.50 \\
139 & 17.78 & 4891 & 2.21 & -0.1 & +0.4 & +0.23 & -0.50 \\
141 & 17.45 & 4977 & 2.42 & -0.6 & +0.4 & -0.36 & +0.12 \\
142 & 17.55 & 5118 & 2.81 & -0.7 & +0.2 & -0.49 & +0.14 \\
201 & 17.54 & 5003 & 2.49 & +0.1 & -0.0 & +0.59 & +0.17 \\
\hline
\end{tabular}




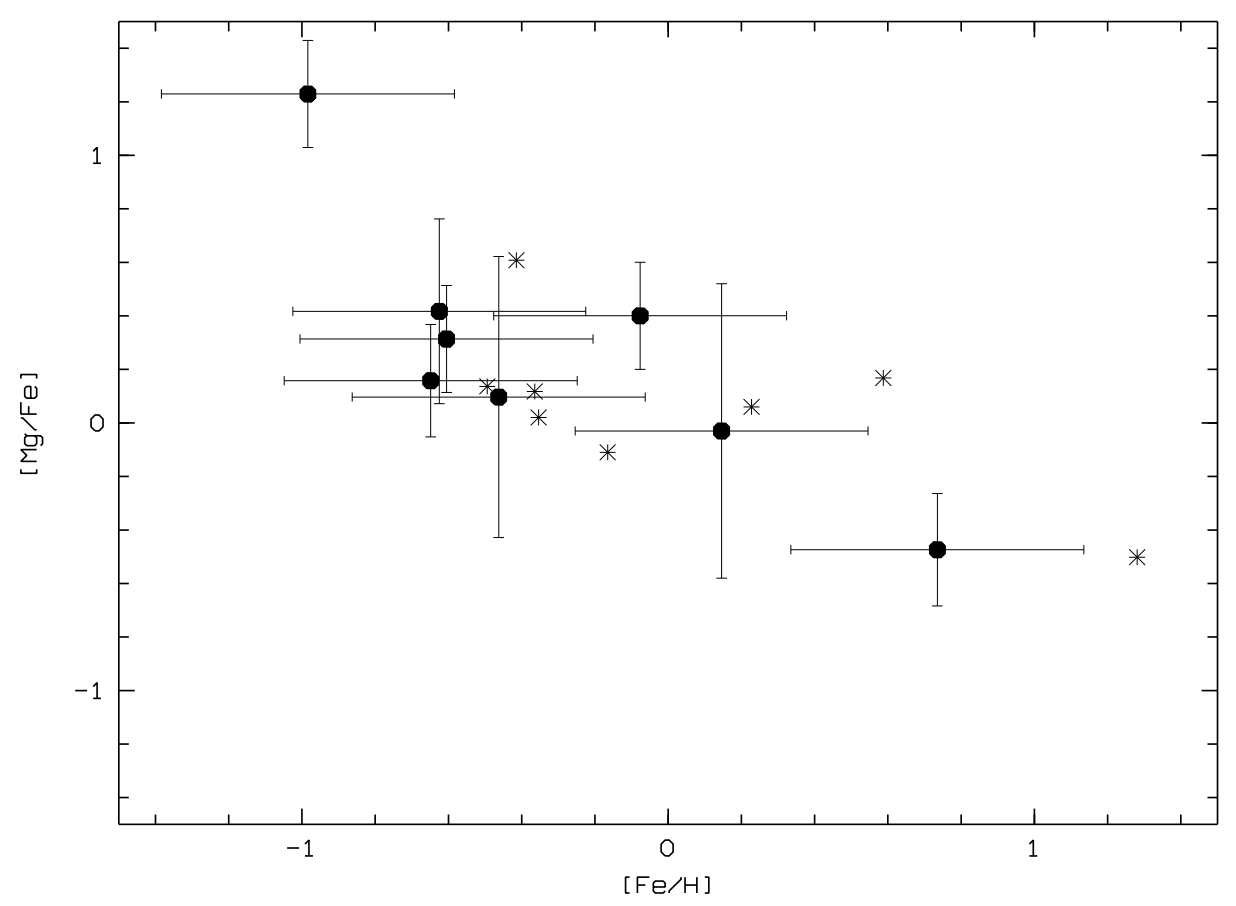

Figure 1. $[\mathrm{Mg} / \mathrm{Fe}]$ ratios versus $[\mathrm{Fe} / \mathrm{H}]$ for $\xi=2.0 \mathrm{kms}^{-1}$ (filled symbols) and $\xi=1.0$ $\mathrm{kms}^{-1}$

\section{Radial velocities and abundances}

The range 480-530 $\mathrm{nm}$ was used to determine heliocentric radial velocities using cross-correlation with synthetic spectra as templates We considered the stars with heliocentric radial velocities in the range $100-180 \mathrm{kms}^{-1}$ to be members of Sagittarius, following Ibata et al (1997), this left us with a sample of 23 stars. In order to estimate abundances we defined six spectral indices which measure the Mgb triplet and some Fe and iron-peak elements features. We developed an iterative procedure which makes use of the SYNTHE code (Kurucz 1993) to determine the abundances which best match the observed to the synthetic indices; details may be found in Marconi et al (1998b). The procedure requires that the atmospheric parameters $\mathrm{T}_{\text {eff }}, \log$ $\mathrm{g}$ and $\xi$ be fixed for each star. The colour $(V-I)_{0}$ of Marconi et al (1998a) was used to determine the effective temperatures using the calibration of Alonso et al (1996); this calibration refers to dwarfs only, however it is theoretically known that the $(V-I)$ colour depends weakly on gravity; we expect the error introduced by neglecting the gravity dependence of $(V-I)$ 
to be on the order of $100 \mathrm{~K}$. The isochrones of Straniero, Chieffi \& Limongi (1997) for an age of $8 \mathrm{Gyr}$ and $[\mathrm{Fe} / \mathrm{H}]=-0.5$ were used to estimate log g. Microturbulence cannot be determined from this intermediate-resolution data, we therefore performed the computations for both $1 \mathrm{kms}^{-1}$ and 2 $\mathrm{kms}^{-1}$, these values cover the range usually found in cool giants and allow to estimate the effect of microturbulence on the derived abundances. This work is still in progress; results for 8 out of the 23 stars with a radial velocity consistent with Sagittarius membership, are presented in Table 1 and displayed in Figure 1.

\section{Discussion}

Our stars have been selected in order to highlight the spread in metallicity derived from the colour-magnitude diagram and in fact among the stars analyzed we see a spread of over one dex. However the metallicities range from super-solar to $[\mathrm{Fe} / \mathrm{H}] \approx-1.0$, about 0.5 dex more metal-rich than the range implied by the photometry. A systematic error in the spectroscopic or photometric (or both!) abundance estimates could reconcile the two results. However closer inspection of figure 1 reveals that of the 8 analyzed stars 3 are solar or super-solar while the remaining 5 show little or no dispersion in metallicity. It is legitimate to ask whether the metal-rich stars belong in fact to the Bulge, in spite of their radial velocity. This hypothesis may not be ruled out, we note however that the $[\mathrm{Mg} / \mathrm{Fe}]$ ratios appear to be solar or sub-solar. The large errors associated with our data preclude any firm conclusion, however, taken at face value, the $[\mathrm{Mg} / \mathrm{Fe}]$ in our metal-rich stars appears to be different from the enhanced ratios displayed by the Bulge $\mathrm{K}$ giants of McWilliam \& Rich (1994), figure 20a. Our findings are in keeping with those of Smecker-Hane, McWilliam \& Ibata (1998), who, on the basis of Keck HIRES spectra, found 2 out of 7 stars to be metal-rich and with $\alpha$ elements $\mathrm{O}$ and $\mathrm{Ca}$ under-abundant with respect to solar.

\section{References}

Alonso A., Arribas S., Martinez-Roger C. 1996, A\& AS, 117, 227

Ibata R.A., Gilmore G., Irwin M.J., 1994, Nat, 370, 194

Ibata R.A., Gilmore G., Irwin M.J., 1995, MNRAS 277, 781

Ibata R.A., Wyse R.F.G., Gilmore G., Irwin M.J., Suntzeff N.B., 1997, AJ, 113, 634

Kurucz R.L., 1993, CD-ROM No. 13, 18

Marconi G., Buonanno R., Castellani M., Iannicola P., Molaro P., Pasquini L., Pulone L., 1998a, A\&A, 330, 453

Marconi G., Bonifacio P., Pasquini L., Molaro P., 1998b in " The Stellar Content of Local Group Galaxies ", held in Cape Town, South Africa, 7-11 September 1998, to be published by ASP, edited by Patricia Whitelock and Russell Cannon, p. 8.

McWilliam A., Rich R.R:, 1994, ApJS, 91, 749

Smecker-Hane T., McWilliam A., Ibata R.A., 1998 AAS Meeting, 1926613

Straniero O., Chieffi A., Limongi M., 1997, ApJ, 490, 425 